OCCASIONAL PIECE

\title{
Lumbar spine region pathology and hamstring and calf injuries in athletes: is there a connection?
}

\author{
J W Orchard, P Farhart, C Leopold
}

Br J Sports Med 2004;38:502-504. doi: 10.1136/bjsm.2003.011346

This paper discusses the theory that subtle lumbosacral canal impingement of the $L 5$ nerve root may be a relatively common occurrence in older footballers and may in fact be a common underlying basis for the age related predisposition towards hamstring and calf strains.

changes occurring at L4/5 and L5/S1 levels, compared with the more proximal levels. The cohort in this study was 31 Olympic athletes aged 19-46, with two thirds in the 20-30 year age group. It is tempting to draw an association between the correlations with the findings related to L5 and S1 levels in table 1 and fig 1.

It is easy to appreciate that an acute disc prolapse at the L5/S1 level may present with hamstring and/or calf pain and limitations in flexibility, which may mimic a muscle strain. There is a further mechanism by which less acute L5/S1 pathology may potentially lead to symptoms (or possibly even strains) in the hamstring and calf muscles. Of all the nerve roots in the lumbosacral plexus, the L5 has the most tortured path through the lumbosacral canal and over the anterior superior ridge of the sacrum, after which it joins the sacral plexus. Briggs and Chandraraj ${ }^{8}$ dissected the lumbosacral ligament (an inconsistent extension of the iliolumbar ligament) in the pelvis, and showed a correlation in cadavers between L5/S1 degenerative changes and compression of the L5 nerve root by the lumbosacral ligament. The cadavers were aged between 60 and 90 years but varied in the relation of lumbosacral ligament to the nerve, with $9 \%$ showing nerve compression and visible flattening of the L5 nerve root by the ligament. ${ }^{8}$ The lumbosacral ligament and its propensity for extraforaminal entrapment of the L5 nerve root was first described by Nathan et $a l^{9}$ and by other anatomists since, ${ }^{10}{ }^{11}$ with extraforaminal entrapment of the L5 nerve root actually first described in $1925 .{ }^{12}$

The lumbosacral ligament, which is present to a degree in everyone, ${ }^{913}$ is visible on MRI scans in certain "normal" patients (using Tl axial scan on a $1.5 \mathrm{~T}$ scanner; fig 2 ) and not in others. It is difficult to assess whether this is because the ligament is atrophic or actually absent in some patients or because the resolution of $1.5 \mathrm{~T}$ scans is not high enough to properly assess the ligament in all patients. Although involving cadavers of average age much greater than that of the average athlete, the study of Briggs and Chandraraj ${ }^{8}$ suggests that the lumbosacral ligament may develop or hypertrophy in response to degenerative changes of the L5/S1 region.

It is possible that the anatomical configuration of a hypertrophied lumbosacral ligament is more clinically significant in older athletes with the common finding of L5/S1 degenerative disc changes. Perhaps, through subtle L5 nerve root entrapment, it is a factor in some of those players who find that they have recurrent hamstring and calf musculotendinous injuries despite regular 
Table 1 Injury prevalence (missed games per team per season) by player age, Australian Football League (reprinted with permission from Sport Health)

\begin{tabular}{llrrrrr}
\hline & & \multicolumn{3}{l}{ Injury prevalence } \\
\cline { 2 - 7 } Nerve supply & Injury category & \multicolumn{1}{l}{$\mathbf{2 1}$} & $\mathbf{2 1 - 2 3}$ & $\mathbf{2 4 - 2 6}$ & $\mathbf{2 7 - 2 9}$ & $\mathbf{3 0 +}$ \\
\hline Local & Lumbar and thoracic spine injuries & 4.5 & 4.0 & 7.2 & 11.5 & 13.3 \\
L1-L4 nerve supply & Groin strains and osteitis pubis & 12.0 & 10.8 & 12.9 & 8.8 & 10.3 \\
& Quadriceps strains & 5.7 & 7.6 & 6.5 & 5.6 & 4.9 \\
& Knee and patella tendon injuries & 1.6 & 2.5 & 2.7 & 1.4 & 3.7 \\
L5-S1 nerve supply & 15.0 & 17.8 & 24.6 & 27.3 & 32.3 \\
& Hamstring strains & 1.8 & 2.2 & 6.5 & 10.1 & 12.0 \\
& Calf strains & 0.5 & 0.5 & 2.7 & 2.2 & 5.5 \\
& Achilles tendon injuries & & & & &
\end{tabular}

preventive maintenance. This hypothesis is interesting as it provides potential additional treatment options to the athlete with recurrent hamstring, calf, and Achilles injuries, which are common in all football codes, track and field, racquet sports, and cricket.

To date we have already had positive experience (although not in the perspective of a controlled study) with imaging guided cortisone injections to the lumbosacral canal region (L5 nerve root) in athletes with peripheral symptoms, such as recurrent hamstring and calf pain. This is a relatively painless and complication free outpatient procedure with quick recovery. We have used this both for acute symptoms and as a preventive procedure in highly susceptible athletes (with a history of multiple muscle strains and pathological changes in the lumbar spine). The limitation of cortisone injections is that any relief from subtle nerve entrapment is only likely to be short term in most cases.

Unfortunately, the lumbosacral ligament is not easily accessible for surgical treatment. The best method for reaching it would probably be through an anterior approach with an abdominal laparoscope. This approach would be a posterolateral abdominal incision anterior to the quadratus lumborum. The lumbosacral canal is found between the iliac vessels and psoas. This type of surgery has recently been described by Matsumato et al..$^{14}$ A posterior approach would be technically easier, but would involve far more surgical trauma of muscles and probably bone (the sacral ala).

It remains to be seen whether it would be technically easy to divide the ligament to free an entrapped L5 nerve root, and whether this procedure would reduce the risk of hamstring or calf injury in a susceptible athlete without causing side effects. There are major possible complications of surgery including potential for damage to the iliac vessels and L5 nerve root itself and potential for L5/S1 segment instability (if the lumbosacral ligament develops as a critical stabiliser of the L5 vertebra).

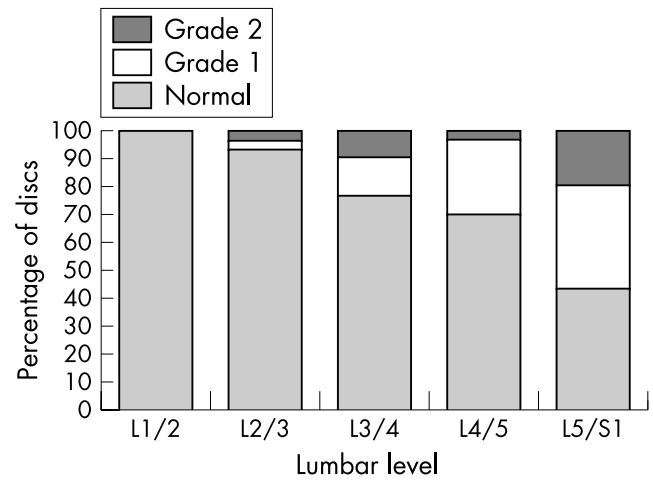

Figure 1 Lumbar disc height. Grade 1, mildly reduced; grade 2, grossly reduced. Reproduced from Ong et af with permission.
Confirming the diagnosis of subtle extraforaminal L5 nerve entrapment, using non-invasive methods, remains problematic. Although MRI scan resolution is improving all the time and can currently isolate structures in the region that may possibly cause entrapment (such as a thick band of the lumbosacral ligament), it probably cannot demonstrate the signs of a nerve entrapment in this region, such as a thinning of the nerve diameter as it passes underneath the ligament. Neurodiagnostic tests may potentially be helpful, but electromyography and nerve conduction changes may only be present after exercise, and even if positive, would have great difficulty in distinguishing between entrapment at the neural foramen, in the lumbosacral canal, or by tight muscles in the gluteal region.

It is most plausible that extraforaminal L5 nerve root entrapment is one of many explanations for a propensity to hamstring and calf symptoms in athletes, in a fashion that is analogous to piriformis syndrome, ${ }^{6}$ sciatic nerve entrapment by the internal obturator muscle, ${ }^{15}$ and hamstring syndrome. ${ }^{16}$ Perhaps multiple subtle entrapments can be present at the same time and be additive, leading to motor dysfunction of the hamstring and calf muscle groups. In a similar fashion to those other previously described syndromes, we believe that the anatomical configuration of lumbosacral ligament entrapment of the L5 nerve root has significant potential to help explain the pathogenesis of posterior thigh and calf injuries in certain athletes. Although this configuration has been well described in the neurological literature for many years, it may also be relevant in the field of sports medicine.

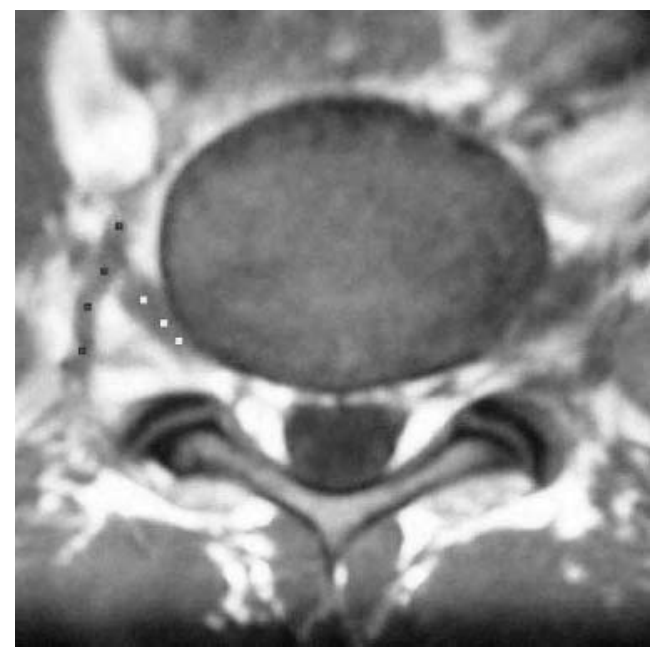

Figure 2 Lumbosacral ligament (marked by four black dots) and its relation to the L5 nerve root (marked by three small white dots) at the level of L5/S1 disc (T1 axial view). 


\section{Authors' affiliations}

J W Orchard, University of Melbourne/South Sydney Sports Medicine, Australia

P Farhart, Cricket New South Wales

C Leopold, Sydney X-Ray

\section{REFERENCES}

1 Orchard J, Seward H. AFL Injury report 2002. Sport Health 2003;21:18-23.

2 Orchard J. Intrinsic and extrinsic risk factors for muscle strains in Australian footballers. Am J Sports Med 2001:300-3.

3 Verrall G, Slavotinek J, Barnes P, et al. Clinical risk factors for hamstring muscle strain injury: a prospective study with correlation of injury by magnetic resonance imaging. Br J Sports Med 2001 ;35:435-40.

4 Orchard J, Marsden J, Lord S, et al. Preseason hamstring muscle weakness associated with hamstring muscle injury in Australian footballers. Am J Sports Med 1997;25:81-5.

5 Bennell $\mathrm{K}$, Wajswelner $\mathrm{H}$, Lew $\mathrm{P}$, et al. Isokinetic strength testing does not predict hamstring injury in Australian Rules footballers. Br J Sports Med 1998;32:309-14.

6 Pecina M, Krmpotic-Nemanic J, Markiewitz A. Piriformis muscle syndrome. In Tunnel syndromes. Peripheral nerve compression syndromes. Boca Raton: CRC Press, 1997: 185-92.

7 Ong A, Anderson J, Roche J. A pilot study of the prevalence of lumbar disc degeneration in elite athletes with lower back pain at the Sydney 2000 Olympic Games. Br J Sports Med 2003;37:263-6.

8 Briggs C, Chandraraj S. Variations in the lumbosacral ligament and associated changes in the lumbosacral region resulting in compression of the fifth dorsal root ganglion and spinal nerve. Clin Anat 1995;8:339-46.

9 Nathan H, Weizenbluth M, Halperin N. The lumbosacral ligament (LSL), with special emphasis on the "lumbosacral tunnel" and the entrapment of the 5th lumbar nerve. Int Orthop 1982;6:197-202.

10 Transfeldt E, Robertson D, Bradford D. Ligaments of the lumbosacral spine and their role in possible extraforaminal spinal nerve entrapment and tethering. J Spinal Disord 1993;6:507-12.
11 Olsewski J, Simmons E, Kallen F, et al. Evidence from cadavers suggestive of entrapment of fifth lumbar spinal nerves by lumbosacral ligaments. Spine 1991; 16:336-47.

12 Danforth $M$, Wilson $P$. The anatomy of the lumbo-sacral region in relation to sciatic pain. J Bone Joint Surg 1925;7:109-60.

13 Pecina M, Krmpotic-Nemanic J, Markiewitz A. Lumbosacral tunnel syndrome. In: Tunnel syndromes. Peripheral nerve compression syndromes. Boca Raton: CRC Press, 1997: 165-7.

14 Matsumoto M, Chiba K, Nojiri K, et al. Extraforaminal entrapment of the fifth lumar spinal nerve by osteophytes of the lumbosacral spine: anatomic study and a report of four cases. Spine 2002;27:E169-73.

15 Meknas K, Christensen A, Johansen $\mathrm{O}$. The internal obturator muscle may cause sciatic pain. Pain 2003; 104:375-80.

16 Orava S. Hamstring syndrome. Operative techniques in sports medicine 1997:5: 143-9.

\section{COMMENTARY}

This brief report prompts us to consider lower lumbar pathology as a source of hamstring and calf problems in people over 30 years of age. Although many of us have thought this way for a while, this is the first evidence to support this concept. This report may raise more questions than it provides answers and certainly invites future studies aimed at risk factor detection and intervention for these kinds of problems.

T M Best

University of Wisconsin Medical School, Madison, WI 53711, USA; tm.best@hosp.wisc.edu 